\title{
ROBUSTNESS OF A TWO-STAGE ESTIMATION PROCEDURE WHEN VARIANCES ARE UNEQUAL
}

\author{
Yoshikazu Takada* and Katuya Miyama*
}

\begin{abstract}
This paper considers properties of the fixed-width confidence interval of the difference of two normal means constructed assuming equal variances when the variances are unequal. We explore the coverage probability of the interval and the sample size. Furthermore, we compare the expected total sample size with that of the fixed width confidence interval constructed without such an assumption.
\end{abstract}

Key words and phrases: Coverage probability, fixed-width confidence interval, sample size, two-stage procedure.

\section{Introduction}

We consider two normal populations $\Pi_{1}$ and $\Pi_{2}$ with means $\mu_{1}$ and $\mu_{2}$ and variances $\sigma_{1}^{2}$ and $\sigma_{2}^{2}$, respectively, where $\mu_{1}, \mu_{2}, \sigma_{1}^{2}$, and $\sigma_{2}^{2}$ are unknown. Having observed $X_{11}, \ldots, X_{1 r}$ from $\Pi_{1}$ and $X_{21}, \ldots, X_{2 s}$ from $\Pi_{2}$, it is required to estimate $\mu_{1}-\mu_{2}$ by $\bar{X}_{1(r)}-\bar{X}_{2(s)}$ within $\pm d$, where $\bar{X}_{1(r)}=\frac{1}{r} \sum_{i=1}^{r} X_{1 i}$, $\bar{X}_{2(s)}=\frac{1}{s} \sum_{i=1}^{s} X_{2 i}$, and $d(>0)$ is a given constant. In order to meet such a requirement, we construct a confidence interval

$$
I_{(r, s)}=\left(\bar{X}_{1(r)}-\bar{X}_{2(s)}-d, \bar{X}_{1(r)}-\bar{X}_{2(s)}+d\right)
$$

with

$$
P\left(\mu_{1}-\mu_{2} \in I_{(r, s)}\right) \geq 1-\alpha
$$

where $1-\alpha(0<\alpha<1)$ is a confidence level. Such an interval is called a fixedwidth confidence interval. It is well known that it is impossible to construct such an interval if the sample sizes $r$ and $s$ are beforehand fixed. For example, see Problem 5.22 of Lehmann and Romano (2005, p. 197). Hence sequential methods are needed to construct such an interval. Two two-stage procedures originated in Stein(1945) were proposed to determine the sample sizes. The first procedure was proposed when the variances are equal, while the second was without such an assumption.

We first consider the procedure assuming equal variances. At the first stage, let $X_{11}, \ldots, X_{1 m}$ and $X_{21}, \ldots, X_{2 m}$ be the observations from populations $\Pi_{1}$ and $\Pi_{2}$ where $m$ is the first sample size. We calculate the sample variances $S_{1(m)}^{2}=\frac{1}{m-1} \sum_{i=1}^{m}\left(X_{1 i}-\bar{X}_{1(m)}\right)^{2}$ and $S_{2(m)}^{2}=\frac{1}{m-1} \sum_{i=1}^{m}\left(X_{2 i}-\bar{X}_{2(m)}\right)^{2}$. Then

\footnotetext{
Received June 20, 2012. Revised August 28, 2012. Accepted September 8, 2012.

*Department of Mathematics and Engineering, Kumamoto University, Kurokami 2-39-1, Kumamoto 860-8555, Japan. Email: takada@kumamoto-u.ac.jp
} 
the total sample size from each population is given by

$$
N=\max \left\{m,\left[\frac{2 a_{m}^{2} U_{m}^{2}}{d^{2}}\right]+1\right\},
$$

where $[u]$ denotes the largest integer less than $u, U_{m}^{2}=\frac{1}{2}\left(S_{1(m)}^{2}+S_{2(m)}^{2}\right)$, and $a_{m}$ denotes the upper $100 \alpha / 2$ percentage point of the $t$ distribution with $2 m-2$ degrees of freedom. If $N>m$, we proceed to the second stage and take additional observation $X_{1 m+1}, \ldots, X_{1 N}$ from $\Pi_{1}$ and $X_{2 m+1}, \ldots, X_{2 N}$ from $\Pi_{2}$. Then

$$
P\left(\mu_{1}-\mu_{2} \in I_{(N, N)}\right) \geq 1-\alpha
$$

when the variances are equal. For example, see Theorem 13.2.1 of Mukhopadhyay and de Silva (2009).

Next we consider the procedure without such an assumption. Based on the first observations $X_{11}, \ldots, X_{1 m}$ and $X_{21}, \ldots, X_{2 m}$, the total sample sizes $N_{1}$ and $N_{2}$ from $\Pi_{1}$ and $\Pi_{2}$ are given by

$$
N_{1}=\max \left\{m,\left[\frac{u_{m} V_{1(m)}\left(V_{1(m)}+V_{2(m)}\right)}{d^{2}}\right]+1\right\}
$$

and

$$
N_{2}=\max \left\{m,\left[\frac{u_{m} V_{2(m)}\left(V_{1(m)}+V_{2(m)}\right)}{d^{2}}\right]+1\right\} \text {, }
$$

where $V_{1(m)}=\sqrt{S_{1(m)}^{2}}$ and $V_{2(m)}=\sqrt{S_{2(m)}^{2}}$. The constant $u_{m}$ is the solution of the equation

$$
2 P\left(\left|T_{m-1}\right|<\sqrt{u}\right)-P\left(\left|T_{m}\right|<\sqrt{\frac{m}{m-1} u}\right)=1-\alpha,
$$

where $T_{v}$ denotes a t random variable with $v$ degrees of freedom. If $N_{i}>m$, we proceed to the second stage and take additional observation $X_{i 1}, \ldots, X_{i N_{i}}$ from $\Pi_{i}, i=1,2$. Then

$$
P\left(\mu_{1}-\mu_{2} \in I_{\left(N_{1}, N_{2}\right)}\right) \geq 1-\alpha .
$$

See Theorem 3 of Schwabe (1995) and Theorem 1 of Takada and Aoshima (1996). Takada (2004) and Aoshima and Yata (2010) considered its extensions to $k$ sample problems. Aoshima et al. (2011) treated the problem from a different perspective.

However, the implementation of the second procedure is troublesome due to the constant $u_{m}$ which is not generally available, though Schwabe (1995) and Takada and Aoshima (1996) provided some values of $u_{m}$ in their tables. Contrary to this, the first procedure is easy to implement because the constant $a_{m}$ is easy to obtain. So it is preferable to use the first procedure if the performance is good even though the variances are unequal.

In this paper, we consider the two-stage procedure assuming equal variances and examine the coverage probability of the fixed-width confidence interval and 
the expected sample size when the variances are unequal. Furthermore, we compare the total sample size between two procedures.

In Section 2, we consider the coverage probability of the fixed-width confidence interval based on the sample size (1.1). In Section 3, we consider the performance of the sample size (1.1) and compare the total sample size with that of the sample sizes (1.2) and (1.3). The comparison of two procedures through an example is considered in Section 4. Section 5 gives brief concluding remarks.

\section{Coverage probability}

In this section, we consider the coverage probability of the fixed-width confidence interval based on the sample size (1.1) when the variances are unequal. We obtain the following lower bound for the coverage probability.

ThEOREM 1. $P\left(\mu_{1}-\mu_{2} \in I_{(N, N)}\right) \geq P\left(\left|T_{m-1}\right|<a_{m}\right)$

Proof. Since the event $\{N=n\}$ and $T_{(n, n)}$ are independent, we have

$$
\begin{aligned}
P\left(\mu_{1}-\mu_{2} \in I_{(N, N)}\right) & =\sum_{n=m}^{\infty} P\left(\left|\bar{X}_{1(n)}-\bar{X}_{2(n)}-\left(\mu_{1}-\mu_{2}\right)\right| \leq d, N=n\right) \\
& =\sum_{n=m}^{\infty} P\left(\left|\bar{X}_{1(n)}-\bar{X}_{2(n)}-\left(\mu_{1}-\mu_{2}\right)\right| \leq d\right) P(N=n) \\
& =\sum_{n=m}^{\infty} G\left(\frac{d^{2} n}{\sigma_{1}^{2}+\sigma_{2}^{2}}\right) P(N=n) \\
& =E\left[G\left(\frac{d^{2} N}{\sigma_{1}^{2}+\sigma_{2}^{2}}\right)\right],
\end{aligned}
$$

where $G(x)$ is the cumulative distribution function of a chi-squared random variable with one degree of freedom. From (1.1)

$$
d^{2} N \geq 2 a_{m}^{2} U_{m}^{2}
$$

Using this inequality in the right hand side of (2.1), we have

$$
P\left(\mu_{1}-\mu_{2} \in I_{(N, N)}\right) \geq E\left[G\left(\frac{2 a_{m}^{2} U_{m}^{2}}{\sigma_{1}^{2}+\sigma_{2}^{2}}\right)\right] .
$$

Writing $Y_{1}=(m-1) S_{1(m)}^{2} / \sigma_{1}^{2}$ and $Y_{2}=(m-1) S_{2(m)}^{2} / \sigma_{2}^{2}$, we have

$$
\begin{aligned}
\frac{2 a_{m}^{2} U_{m}^{2}}{\sigma_{1}^{2}+\sigma_{2}^{2}} & =\frac{a_{m}^{2}\left(\sigma_{1}^{2} Y_{1}+\sigma_{2}^{2} Y_{2}\right)}{(m-1)\left(\sigma_{1}^{2}+\sigma_{2}^{2}\right)} \\
& =\frac{\sigma_{1}^{2}}{\sigma_{1}^{2}+\sigma_{2}^{2}}\left(\frac{a_{m}^{2} Y_{1}}{m-1}\right)+\frac{\sigma_{2}^{2}}{\sigma_{1}^{2}+\sigma_{2}^{2}}\left(\frac{a_{m}^{2} Y_{2}}{m-1}\right),
\end{aligned}
$$

so that

$$
G\left(\frac{2 a_{m}^{2} U_{m}^{2}}{\sigma_{1}^{2}+\sigma_{2}^{2}}\right) \geq \frac{\sigma_{1}^{2}}{\sigma_{1}^{2}+\sigma_{2}^{2}} G\left(\frac{a_{m}^{2} Y_{1}}{m-1}\right)+\frac{\sigma_{2}^{2}}{\sigma_{1}^{2}+\sigma_{2}^{2}} G\left(\frac{a_{m}^{2} Y_{2}}{m-1}\right) .
$$


The last inequality follows from the fact that $-G(x)$ is convex. Since $Y_{1}$ and $Y_{2}$ are chi-squared random variables with $m-1$ degrees of freedom, it follows from (2.2) that

$$
P\left(\mu_{1}-\mu_{2} \in I_{(N, N)}\right) \geq E\left[G\left(\frac{a_{m}^{2} Y_{1}}{m-1}\right)\right]=P\left(\left|T_{m-1}\right|<a_{m}\right),
$$

which proves the result.

Table 1 gives numerical values of the lower bound (LB) for $m=10(2) 30$ and $1-\alpha=0.95$. The table shows that the coverage probability is not so bad compared with the nominal value if we properly choose the first sample size. For example, when the nominal value is $95 \%$, if we choose 20 observations as the first sample, it is guaranteed that the coverage probability is at least greater than $94.3 \%$ even though the variances are unequal. The following result shows that the lower bound is exact.

Table 1. The lower bound of the coverage probability when $1-\alpha=0.95$.

\begin{tabular}{r|ccccccccccc}
\hline$m$ & 10 & 12 & 14 & 16 & 18 & 20 & 22 & 24 & 26 & 28 & 30 \\
\hline LB & 0.935 & 0.937 & 0.939 & 0.941 & 0.942 & 0.943 & 0.943 & 0.944 & 0.944 & 0.945 & 0.945 \\
\hline
\end{tabular}

THEOREM 2. If one of the variances is fixed and the other goes to infinity, then

$$
\lim P\left(\mu_{1}-\mu_{2} \in I_{(N, N)}\right)=P\left(\left|T_{m-1}\right|<a_{m}\right) .
$$

Proof. Assume that $\sigma_{1}^{2}$ is fixed and $\sigma_{2}^{2}$ goes to infinity. It follows from (1.1) that

$$
\frac{2 a_{m}^{2} U_{m}^{2}}{\sigma_{1}^{2}+\sigma_{2}^{2}}<\frac{d^{2} N}{\sigma_{1}^{2}+\sigma_{2}^{2}}<\frac{2 a_{m}^{2} U_{m}^{2}}{\sigma_{1}^{2}+\sigma_{2}^{2}}+\frac{m d^{2}}{\sigma_{1}^{2}+\sigma_{2}^{2}}
$$

Hence from (2.3)

$$
\begin{aligned}
\frac{a_{m}^{2}}{m-1}\left(\frac{1}{1+r} Y_{1}+\frac{r}{1+r} Y_{2}\right)<\frac{d^{2} N}{\sigma_{1}^{2}+\sigma_{2}^{2}}< & \frac{a_{m}^{2}}{m-1}\left(\frac{1}{1+r} Y_{1}+\frac{r}{1+r} Y_{2}\right) \\
& +\frac{m a_{m}^{2}}{\lambda}
\end{aligned}
$$

where $r=\sigma_{2}^{2} / \sigma_{1}^{2}$ and $\lambda=a_{m}^{2}\left(\sigma_{1}^{2}+\sigma_{2}^{2}\right) / d^{2}$, so that as $\sigma_{2}^{2}$ goes to infinity, we have

$$
\lim \frac{d^{2} N}{\sigma_{1}^{2}+\sigma_{2}^{2}}=\frac{a_{m}^{2}}{m-1} Y_{2}
$$

Then the result follows from (2.1).

Since $P\left(\left|T_{m-1}\right|<a_{m}\right)<1-\alpha$, Theorem 2 implies that the coverage probability become less than the nominal value when the difference between the variances 
becomes large. In order to further explore the coverage probability, we need the following lemma, the proof of which is immediately obtained from (2.1) and (2.4).

LEMMA 1. If $\sigma_{1}^{2}$ and $\sigma_{2}^{2}$ go to infinity with $r=\sigma_{2}^{2} / \sigma_{1}^{2}$ fixed, then

$$
\begin{aligned}
& \lim P\left(\mu_{1}-\mu_{2} \in I_{(N, N)}\right) \\
& \quad=E\left[G\left(\frac{1}{1+r}\left(\frac{a_{m}^{2} Y_{1}}{m-1}\right)+\frac{r}{1+r}\left(\frac{a_{m}^{2} Y_{2}}{m-1}\right)\right)\right],
\end{aligned}
$$

where $Y_{1}$ and $Y_{2}$ are independent chi-squared random variables with $m-1$ degrees of freedom.

Remark. The result of Lemma 1 (and Theorems in the next section) also follows from the condition that $d$ goes to zero with $\sigma_{1}^{2}$ and $\sigma_{2}^{2}$ fixed.

Let the right hand side of (2.5) be

$$
F(\omega)=E\left[G\left(\frac{a_{m}^{2}}{m-1} Y\right)\right]
$$

where $Y=\omega Y_{1}+(1-\omega) Y_{2}$ with $\omega=1 /(1+r)$. We approximate the distribution of $Y$ by that of $c \chi_{v}^{2}$ where $\chi_{v}^{2}$ is a chi-squared random variable with $v$ degrees of freedom and $c$ is a positive constant, which is known as Satterthwaite's method. Then

$$
c=\omega^{2}+(1-\omega)^{2}, \quad v=\frac{m-1}{c} .
$$

See the Appendix for the derivation of (2.7). Hence from (2.6)

$$
F(\omega) \cong E\left[G\left(\frac{a_{m}^{2} \chi_{v}^{2}}{v}\right)\right]=P\left(\left|T_{v}\right|<a_{m}\right) .
$$

From the property of the t-distribution, the right hand side of (2.8) is maximized when $\omega=1 / 2$, which corresponds to the equal variances. Hence it is anticipated that the coverage probability is maximized when the variances are equal. In order to confirm this anticipation, we conducted simulations. We chose that $m=10, d=1.0$, and $1-\alpha=0.95$. Table 2 gives the simulation results based on 10,000 replications for $\sigma_{1}^{2}+\sigma_{2}^{2}=10$ and 20, in which CP denotes the estimates of the coverage probability. The simulation results seem to support what was anticipated above.

Table 2. Estimates of the coverage probability $(m=10, d=1.0,1-\alpha=0.95)$.

\begin{tabular}{c|ccccc}
\multicolumn{7}{c}{$\sigma_{1}^{2}+\sigma_{2}^{2}=10$} \\
\hline$\left(\sigma_{1}^{2}, \sigma_{2}^{2}\right)$ & $(2,8)$ & $(4,6)$ & $(5,5)$ & $(6,4)$ & $(8,2)$ \\
\hline $\mathrm{CP}$ & 0.944 & 0.950 & 0.952 & 0.950 & 0.948 \\
\hline \multicolumn{7}{c}{$\sigma_{1}^{2}+\sigma_{2}^{2}=20$} \\
\hline$\left(\sigma_{1}^{2}, \sigma_{2}^{2}\right)$ & $(4,16)$ & $(8,12)$ & $(10,10)$ & $(12,8)$ & $(16,4)$ \\
\hline $\mathrm{CP}$ & 0.947 & 0.951 & 0.953 & 0.950 & 0.947 \\
\hline
\end{tabular}




\section{Sample size}

In this section, we evaluate the expected sample size of each procedure and compare the total sample sizes. In order to derive the expected sample size, we need the following lemma, which is maybe well known, but for the completeness the proof is given in the Appendix.

LEMMA 2. Let $X$ be a non-negative random variable with a density function. Then

$$
\lim _{\lambda \rightarrow \infty} E(\lambda X-[\lambda X])=\frac{1}{2}
$$

We first give an asymptotic evaluation of $E(N)$.

ThEOREM 3. If $\sigma_{1}$ and $\sigma_{2}$ go to infinity with $\phi=\sigma_{2} / \sigma_{1}$ fixed, then

$$
\lim E(N-\lambda)=\frac{1}{2}
$$

where $\lambda=\frac{a_{m}^{2}\left(\sigma_{1}^{2}+\sigma_{2}^{2}\right)}{d^{2}}$.

Proof. Let $R=\left[\frac{2 a_{m}^{2} U_{m}^{2}}{d^{2}}\right]+1$ and $J=\frac{2 a_{m}^{2} U_{m}^{2}}{d^{2}}-\left[\frac{2 a_{m}^{2} U_{m}^{2}}{d^{2}}\right]$. Then

$$
R=\frac{2 a_{m}^{2} U_{m}^{2}}{d^{2}}-J+1
$$

so that

$$
E(R)=\lambda-E(J)+1 .
$$

Since $N=\max (m, R)$,

$$
0 \leq E(N-R) \leq m P(N=m) .
$$

It follows from (1.1) and (2.3) that

$$
P(N=m)=P\left(\frac{2 a_{m}^{2} U_{m}^{2}}{d^{2}} \leq m\right)=P\left(\frac{1}{m-1}\left(\omega Y_{1}+(1-\omega) Y_{2}\right) \leq \frac{m}{\lambda}\right)
$$

where $\omega=1 /\left(1+\phi^{2}\right)$. Hence

$$
\lim P(N=m)=0,
$$

so that from (3.2)

$$
\lim E(N-R)=0,
$$

which implies

$$
\lim E(N-\lambda)=\lim E(R-\lambda) .
$$

Since

$$
\frac{2 a_{m}^{2} U_{m}^{2}}{d^{2}}=\frac{\lambda\left(\omega Y_{1}+(1-\omega) Y_{2}\right)}{m-1}
$$


it follows from Lemma 2 that

$$
\lim E(J)=\frac{1}{2}
$$

so that from (3.1) and (3.3)

$$
\lim E(N-\lambda)=\frac{1}{2}
$$

which completes the proof.

From Theorem 3 we can approximate the value of $E(N)$ by $\lambda+1 / 2$ for large $\sigma_{1}^{2}$ and $\sigma_{2}^{2}$. In order to see how good this approximation is, we conducted simulations when $m=10, d=1.0$, and $1-\alpha=0.95$ for $\sigma_{1}^{2}+\sigma_{2}^{2}=10$ and 20 . Table 3 gives the estimates of $E(N)$ and its standard error (SE) based on 10,000 replications.

Table 3. Estimates of $\mathrm{E}(\mathrm{N})(m=10, d=1.0,1-\alpha=0.95)$.

\begin{tabular}{c|ccccc|c}
\multicolumn{7}{c}{$\sigma_{1}^{2}+\sigma_{2}^{2}=10$} \\
\hline$\left(\sigma_{1}^{2}, \sigma_{2}^{2}\right)$ & $(2,8)$ & $(4,6)$ & $(5,5)$ & $(6,4)$ & $(8,2)$ & $\lambda+\frac{1}{2}$ \\
\hline $\mathrm{E}(\mathrm{N})$ & 44.5 & 44.7 & 44.4 & 44.5 & 44.6 & 44.6 \\
$\mathrm{SE}$ & 0.17 & 0.15 & 0.15 & 0.15 & 0.17 & \\
\hline
\end{tabular}

\begin{tabular}{c|ccccc|c}
\multicolumn{7}{c}{$\sigma_{1}^{2}+\sigma_{2}^{2}=20$} \\
\hline$\left(\sigma_{1}^{2}, \sigma_{2}^{2}\right)$ & $(4,16)$ & $(8,12)$ & $(10,10)$ & $(12,8)$ & $(16,4)$ & $\lambda+\frac{1}{2}$ \\
\hline $\mathrm{E}(\mathrm{N})$ & 88.9 & 88.5 & 88.5 & 88.6 & 88.9 & 88.8 \\
$\mathrm{SE}$ & 0.35 & 0.30 & 0.29 & 0.30 & 0.35 & \\
\hline
\end{tabular}

From Table 3 it turns out that $E(N)$ seems to depend on $\sigma_{1}^{2}$ and $\sigma_{2}^{2}$ through $\sigma_{1}^{2}+\sigma_{2}^{2}$, and $\lambda+\frac{1}{2}$ gives a good approximation to $E(N)$.

The next result gives the asymptotic evaluations of $E\left(N_{1}\right)$ and $E\left(N_{2}\right)$, the proof of which is given in the Appendix.

TheOREM 4. It $\sigma_{1}$ and $\sigma_{2}$ go to infinity with $\phi=\sigma_{2} / \sigma_{1}$ fixed, Then

$$
\lim E\left(N_{1}-\lambda_{1}\right)=\frac{1}{2}, \quad \lim E\left(N_{2}-\lambda_{2}\right)=\frac{1}{2},
$$

where $\lambda_{1}=\frac{u_{m} \sigma_{1}^{2}}{d^{2}}\left(1+\phi \rho^{2}\right)$ and $\lambda_{2}=\frac{u_{m} \sigma_{2}^{2}}{d^{2}}\left(1+\frac{\rho^{2}}{\phi}\right)$ with $\rho=\sqrt{\frac{2}{m-1}} \frac{\Gamma\left(\frac{m}{2}\right)}{\Gamma\left(\frac{m-1}{2}\right)}$, and $\Gamma(x)$ is the gamma function.

In order to see how good the approximations $\lambda_{1}+1 / 2$ and $\lambda_{2}+1 / 2$ to $E\left(N_{1}\right)$ and $E\left(N_{2}\right)$ are, we conducted simulations when $m=15(1) 25, d=1.0$, $1-\alpha=0.95, \sigma_{1}=3.0$, and $\sigma_{2}=1.5$. Table 4 gives the results. Table 4 shows that the approximations $\lambda_{1}+1 / 2$ and $\lambda_{2}+1 / 2$ of $E\left(N_{1}\right)$ and $E\left(N_{2}\right)$ are fairly good. 
Table 4. Estimates of $E\left(N_{1}\right)$ and $E\left(N_{2}\right)\left(d=1.0,1-\alpha=0.95, \sigma_{1}=3.0, \sigma_{2}=1.5\right)$.

\begin{tabular}{c|cc|cc|cc}
\hline$m$ & $E\left(N_{1}\right)$ & $\mathrm{SE}$ & $E\left(N_{2}\right)$ & $\mathrm{SE}$ & $\lambda_{1}+1 / 2$ & $\lambda_{2}+1 / 2$ \\
\hline 15 & 66.4 & 0.21 & 33.2 & 0.09 & 66.6 & 33.2 \\
16 & 65.3 & 0.20 & 32.7 & 0.09 & 65.5 & 32.6 \\
17 & 64.3 & 0.20 & 32.0 & 0.08 & 64.6 & 32.2 \\
18 & 63.5 & 0.18 & 31.8 & 0.08 & 63.7 & 31.8 \\
19 & 63.2 & 0.18 & 31.5 & 0.08 & 63.0 & 31.5 \\
20 & 62.5 & 0.17 & 31.1 & 0.07 & 62.4 & 31.2 \\
21 & 61.6 & 0.16 & 31.0 & 0.07 & 61.8 & 30.9 \\
22 & 61.2 & 0.16 & 30.9 & 0.07 & 61.3 & 30.7 \\
23 & 60.9 & 0.15 & 30.9 & 0.06 & 60.9 & 30.5 \\
24 & 60.5 & 0.15 & 30.7 & 0.06 & 60.5 & 30.3 \\
25 & 59.9 & 0.15 & 30.7 & 0.06 & 60.1 & 30.1 \\
\hline
\end{tabular}

Table 5. The interval of $\phi=\sigma_{2} / \sigma_{1}$ in which $\lambda_{1}+\lambda_{2}-2 \lambda>0(1-\alpha=0.95)$.

\begin{tabular}{c|ccccccccccc}
\hline$m$ & 15 & 16 & 17 & 18 & 19 & 20 & 21 & 22 & 23 & 24 & 25 \\
\hline$\phi_{L}$ & 0.42 & 0.44 & 0.45 & 0.47 & 0.48 & 0.49 & 0.50 & 0.51 & 0.52 & 0.53 & 0.54 \\
$\phi_{U}$ & 2.36 & 2.27 & 2.20 & 2.13 & 2.07 & 2.02 & 1.98 & 1.94 & 1.91 & 1.88 & 1.85 \\
\hline
\end{tabular}

The following result gives the comparison of total sample sizes between two procedures, which is easy to follow from Theorems 3 and 4.

THEOREM 5. It $\sigma_{1}$ and $\sigma_{2}$ go to infinity with $\phi=\sigma_{2} / \sigma_{1}$ fixed, then

$$
\lim E\left(N_{1}+N_{2}-2 N-\left(\lambda_{1}+\lambda_{2}-2 \lambda\right)\right)=0
$$

and

$$
\lambda_{1}+\lambda_{2}-2 \lambda=\frac{\sigma_{1}^{2}}{d^{2}} f(\phi)
$$

where

$$
f(\phi)=\left(u_{m}-2 a_{m}^{2}\right) \phi^{2}+2 u_{m} \rho^{2} \phi-2 a_{m}^{2}
$$

Theorem 5 shows that $E\left(N_{1}+N_{2}\right)-2 E(N)$ is approximated by $\lambda_{1}+\lambda_{2}-2 \lambda$. Table 5 gives the interval $\left(\phi_{L}, \phi_{U}\right)$ of $\phi$ in which $\lambda_{1}+\lambda_{2}-2 \lambda>0$ for $m=15(1) 25$ and $1-\alpha=0.95$. Each interval in Table 5 contains one corresponding to the equal variances, which shows that when the difference between the variances is small (large) the procedure assuming equal variances needs fewer (more) observations than the procedure without such an assumption.

\section{Example}

We use the data of Example 2 in Aoshima and Mukhopadhyay (2002) to see the performance of two procedures when applied to real data. The example concerns two organic diets (diet 1 and diet 2) fed to raise a large number of chicks in a poultry farm. The weight (ounce) of a chick is approximately distributed 
Table 6. $\operatorname{diet} 1$.

\begin{tabular}{llllllllll}
\hline 81.2 & 71.6 & 80.5 & 78.5 & 89.5 & 81.3 & 81.0 & 84.9 & 82.2 & 82.6 \\
77.9 & 82.4 & 79.8 & 78.1 & 79.3 & 82.5 & 84.1 & 77.6 & 82.6 & 78.9 \\
\hline
\end{tabular}

Table 7 . diet 2 .

\begin{tabular}{llllllllll}
\hline 79.7 & 81.9 & 81.7 & 88.9 & 79.8 & 77.6 & 88.9 & 84.6 & 84.8 & 82.0 \\
82.0 & 83.3 & 77.9 & 71.1 & 81.2 & 78.1 & 76.1 & 83.0 & 86.8 & 77.0 \\
\hline
\end{tabular}

according to a normal distribution. We are interested in the problem of estimating the difference of the population means by a fixed-width confidence interval with $d=1.5$ and $1-\alpha=0.95$. We take the first sample size $m$ equal to 20 . Tables 6 and 7 are the data of the first 20 observations from Tables 10 (diet 1) and 11 (diet 2) in Aoshima and Mukhopadhyay (2002). 19.32

We first consider the sample size (1.1). Since $S_{1(20)}^{2}=12.66$ and $S_{2(20)}^{2}=$

$$
U_{20}^{2}=\frac{1}{2}(12.66+19.32)=15.99
$$

Substituting $m=20, d=1.5, a_{20}=2.024$ and $U_{20}=15.99$ into (1.1), we have

$$
N=\max \left\{20,\left[\frac{2 \times 2.024^{2} \times 15.99}{1.5^{2}}\right]+1\right\}=59 .
$$

Hence $N-m=59-20=39$, so that 39 additional observations from each population are needed. We took the next 39 observations from Tables 10 and 11 in Aoshima and Mukhopadhyay (2002). Then

$$
\bar{X}_{1(59)}=80.94, \quad \bar{X}_{2(59)}=81.51,
$$

so that $T_{(51,51)}=80.94-81.51=-0.57$, and the fixed-width confidence interval becomes

$$
(-0.57-1.5,-0.57+1.5)=(-2.07,0.93) .
$$

Next we consider the sample sizes (1.2) and (1.3) using Tables 6 and 7 as the first sample observations. From Table 1 in Schwabe (1995), we find $u_{20}=2.15^{2}$. Since $V_{1(20)}=\sqrt{S_{1(20)}^{2}}=3.56$ and $V_{2(20)}=\sqrt{S_{2(20)}^{2}}=4.40$, it follows from (1.2) and (1.3) that

$$
N_{1}=\max \left\{20,\left[\frac{2.15^{2} \times 3.56 \times(3.56+4.40)}{1.5^{2}}\right]+1\right\}=59
$$

and

$$
N_{2}=\max \left\{20,\left[\frac{2.15^{2} \times 4.40 \times(3.56+4.40)}{1.5^{2}}\right]+1\right\}=72 .
$$

Hence $N_{1}-m=59-20=39$ and $N_{2}-m=72-20=52$, so that we took the next 39 observations from Table 10 and 52 observations from Table 11 in 
Aoshima and Mukhopadyay (2002). Then

$$
\bar{X}_{1(59)}=80.94, \quad \bar{X}_{2(72)}=81.43,
$$

so that $T_{(59,72)}=80.94-81.43=-0.49$ and the fixed-width confidence interval becomes

$$
(-0.49-1.5,-0.49+1.5)=(-1.99,1.01) .
$$

Now let us compare the total sample sizes between the two procedures. Since $2 N=2 \times 59=118$ and $N_{1}+N_{2}=59+72=131$, the total sample size of the procedure assuming equal variances is less than that of the procedure without such an assumption. One reason is that based on the first stage observations, the estimate of $\sigma_{1}$ is $V_{1(20)}=3.56$ and that of $\sigma_{2}$ is $V_{2(20)}=4.40$, so that the estimate of $\phi=\sigma_{2} / \sigma_{1}$ is $4.40 / 3.56=1.24$, which is included in the interval of Table 5 . The other reason is that the $\mathrm{P}$-value of the hypothesis test regarding equal variances based on the first stage observations is 0.365 , so that the difference between the variances are considered small.

\section{Concluding remarks}

We considered the performance of the coverage probability and the expected sample size of the two-stage procedure assuming equal variances when the variances are unequal. It turns out that the reduction of the coverage probability is permissible compared with the nominal value for a moderate first sample size, and that the expected sample size is superior to that of the procedure without such an assumption when the difference between the variances is not large. So the choice between two procedures depends on the difference between the variances, which leads us to consider the following hybrid two-stage procedure. At the first stage, we perform the hypothesis test regarding the equal variances based on the first stage observations. If the hypothesis is not rejected, we use the procedure assuming equal variances in the second stage, otherwise, we use the procedure without such an assumption in the second stage. In the near future we will study the performance of this hybrid procedure. Furthermore, we will extend the results to the $k$-sample problem.

\section{Appendix}

ProOF OF (2.7). It is easy to see that

$$
E(Y)=m-1, \quad V(Y)=2(m-1)\left(\omega^{2}+(1-\omega)^{2}\right) .
$$

On the other hand,

$$
E\left(c \chi_{v}^{2}\right)=c v, \quad V\left(c \chi_{v}^{2}\right)=2 c^{2} v
$$

Hence

$$
m-1=c v, \quad 2(m-1)\left(\omega^{2}+(1-\omega)^{2}\right)=2 c^{2} v,
$$

which gives

$$
c=\omega^{2}+(1-\omega)^{2}, \quad v=\frac{m-1}{\omega^{2}+(1-\omega)^{2}}=\frac{m-1}{c} .
$$


Proof of Lemma 2. Let $U=\lambda x-[\lambda x]$. It is enough to show that $U$ converges to a uniform distribution on $(0,1)$ as $\lambda \rightarrow \infty$. Let $G(x)$ and $g(x)$ be the cumulative distribution function and the density function of $X$. Then for $0<u<1$

$$
\begin{aligned}
P(U \leq u) & =P(\lambda X-[\lambda X] \leq u) \\
& =\sum_{n=0}^{\infty} P(\lambda X-[\lambda X] \leq u, \quad[\lambda X]=n) \\
& =\sum_{n=0}^{\infty} P(\lambda X-n \leq u, n \leq \lambda X<n+1) \\
& =\sum_{n=0}^{\infty} P(n \leq \lambda X \leq n+u) \\
& =\sum_{n=0}^{\infty}\left(G\left(\frac{n+u}{\lambda}\right)-G\left(\frac{n}{\lambda}\right)\right) \\
& =\sum_{n=0}^{\infty} \frac{u}{\lambda} g\left(\xi_{n}\right),
\end{aligned}
$$

where $n / \lambda \leq \xi_{n} \leq(n+u) / \lambda(<(n+1) / \lambda)$. Hence

$$
\begin{aligned}
\lim _{\lambda \rightarrow \infty} P(U \leq u) & =u \lim _{\lambda \rightarrow \infty} \sum_{n=0}^{\infty} \frac{1}{\lambda} g\left(\xi_{n}\right) \\
& =u \int_{0}^{\infty} g(x) d x \\
& =u,
\end{aligned}
$$

which completes the proof.

Proof of Theorem 4. We only show the result for $N_{1}$, because the proof for $N_{2}$ is similar. Let $W_{1}=\sqrt{m-1} V_{1(m)} / \sigma_{1}$ and $W_{2}=\sqrt{m-1} V_{2(m)} / \sigma_{2}$. Then $W_{1}^{2}$ and $W_{2}^{2}$ are independent chi-squared random variables with $m-1$ degrees of freedom, and

$$
\frac{u_{m} V_{1 m}\left(V_{1 m}+V_{2 m}\right)}{d^{2}}=\sigma_{1}^{2} \frac{u_{m}\left(W_{1}^{2}+\phi W_{1} W_{2}\right)}{(m-1) d^{2}},
$$

which yields

$$
\begin{aligned}
E\left(\frac{u_{m} V_{1(m)}\left(V_{1(m)}+V_{2(m)}\right)}{d^{2}}\right) & =\frac{\sigma_{1}^{2} u_{m}}{(m-1) d^{2}}\left(m-1+\phi E\left(W_{1}\right)^{2}\right) \\
& =\frac{\sigma_{1}^{2} u_{m}}{d^{2}}\left\{1+\phi\left(\frac{E\left(W_{1}\right)}{\sqrt{m-1}}\right)^{2}\right\} \\
& =\frac{\sigma_{1}^{2} u_{m}}{d^{2}}\left(1+\phi \rho^{2}\right),
\end{aligned}
$$


since $E\left(W_{1}\right) / \sqrt{m-1}=\rho$. Letting

$$
R_{1}=\left[\frac{u_{m} V_{1 m}\left(V_{1 m}+V_{2 m}\right)}{d^{2}}\right]+1
$$

and

$$
J_{1}=\frac{u_{m} V_{1 m}\left(V_{1 m}+V_{2 m}\right)}{d^{2}}-\left[\frac{u_{m} V_{1 m}\left(V_{1 m}+V_{2 m}\right)}{d^{2}}\right]
$$

we have

$$
R_{1}=\frac{u_{m} V_{1 m}\left(V_{1 m}+V_{2 m}\right)}{d^{2}}-J_{1}+1,
$$

so that from (A.2)

$$
E\left(R_{1}\right)=\lambda_{1}-E\left(J_{1}\right)+1 .
$$

From $(1.2), N_{1}=\max \left(m, R_{1}\right)$. Hence

$$
0 \leq E\left(N_{1}-R_{1}\right) \leq m P\left(N_{1}=m\right) .
$$

It follows from (A.1) that

$$
P\left(N_{1}=m\right)=P\left(\frac{u_{m}\left(W_{1}^{2}+\phi W_{1} W_{2}\right)}{(m-1) d^{2}} \leq \frac{m}{\sigma_{1}^{2}}\right),
$$

which implies that $\lim P\left(N_{1}=m\right)=0$, so that from (A.4)

$$
\lim E\left(N_{1}-R_{1}\right)=0
$$

and hence

$$
\lim E\left(N_{1}-\lambda_{1}\right)=\lim E\left(R_{1}-\lambda_{1}\right)
$$

It follows from Lemma 3.1 and (A.1)

$$
\lim E\left(J_{1}\right)=\frac{1}{2}
$$

so that from (A.3) and (A.5)

$$
\lim E\left(N_{1}-\lambda_{1}\right)=\frac{1}{2}
$$

which completes the proof.

\section{Acknowledgements}

The authors thank the Associate Editor and the referee for their valuable comments. The work was supported by Grant-in-Aid for Scientific Research (21540138) of Japan Society for the Promotion of Science. 


\section{REFERENCES}

Aoshima, M. and Mukhopadhyay, N. (2002). Two-stage estimation of a linear function of normal means with second-order approximations, Sequential Analysis, 21, 109-144.

Aoshima, M. and Yata, K. (2010). Asymptotic second-order consistency for two-stage estimation methodologies and its applications, Ann. Inst. Stat. Math., 62, 571-600.

Aoshima, M., Mukhopadhyay, N. and Kobayashi, Y. (2011). Two-stage procedures for estimating the difference of means when the sampling cost is different, Sequential Analysis, 30, $160-171$.

Lehmann, E. L. and Romano, J. P. (2005). Testing Statistical Hypotheses, Third edition, Springer-Verlag, New York.

Mukhopadhyay, N. and de Silva, B. M. (2009). Sequential Methods and Their Applications, CRC Press, New York.

Schwabe, R. (1995). Some two-stage procedures for treating the Behrens-Fisher problem, ModelOriented Data Analysis (eds. W. G. Müller, H. P. Wynn and A. A. Zhigljavsky), SpringerVerlag, New York, 81-89.

Stein, C. (1945). A two sample test for a linear hypothesis whose power is independent of the variance, Ann. Math. Statist., 16, 243-258.

Takada, Y. (2004). Asymptotic second-order efficiency of a two-stage procedure for estimating a linear function of normal means, Sequential Analysis, 25, 103-120.

Takada, Y. and Aoshima, M. (1996). Two-stage procedures for the difference of two multinormal means with covariance matrices different only by unknown scalar, Commun. Statist. Theor. Math., 25, 2371-2379. 